\title{
TRATAMENTO FISIOTERAPÊUTICO EM PRATICANTES DE ATIVIDADE FÍSICA COM INCONTINÊNCIA URINÁRIA
}

\author{
Larissa Bianchi Ilhéu, Kelley Cristina Coelho, Daniela Saldanha Wittig e Siméia \\ Gaspar Palácio \\ UNICESUMAR \\ larissailheu@gmail.com
}

Acesso DOI: http://dx.doi.org/10.34059/ciejop.2019v27i1-18

\section{RESUMO}

Introdução: A incontinência urinária é caracterizada pela perda involuntária de urina, podendo ocorrer em diversas situações. O tipo mais comum é a incontinência urinária de esforço, que associada à prática de atividade física afeta a pressão intraabdominal e causa fraqueza do assoalho pélvico. O objetivo principal desta pesquisa foi avaliar a eficácia da fisioterapia pélvica como forma de tratamento para incontinência urinária de esforço. Metodologia: A pesquisa foi realizada na Clínica de Fisioterapia da Unicesumar. Participaram desta pesquisa 2 voluntárias do sexo feminino com diagnóstico de incontinência urinária de esforço, praticantes de atividade física, com idade em média de 40 anos. As pacientes foram submetidas a 10 sessões de fisioterapia pélvica utilizando a técnica da ginástica hipopressiva, duas vezes por semana no período de 45 minutos. Os resultados encontrados mostram que o tratamento fisioterapêutico utilizando a ginástica hipopressiva, pode promover o fortalecimento do assoalho pélvico, e desta forma melhorar a qualidade de vida dos indivíduos pesquisados e diminuir o quadro de incontinência urinária em praticantes de atividade física.

PALAVRAS-CHAVE: Incontinência urinaria, fisioterapia uroginecológica, atividade física, ginástica hipopressiva, assoalho pélvico.

\section{ABSTRACT}

Introduction: Urinary incontinence is characterized by the involuntary loss of urine, which can occur in several situations. The most common type is stress urinary incontinence, which associated with physical activity practice, affects intra-abdominal pressure and weakness of the pelvic floor. The main objective of this research was to evaluate the effectiveness of physical therapy as a form of treatment for stress urinary incontinence. Methodology: The research was conducted at the Physiotherapy clinic of Unicesumar University. Two volunteers with a diagnosis of stress urinary incontinence, physical activity practitioners, with a mean age of 40 years, were included in this study. The patients were submitted to the treatment using the hypopressive gymnastic technique, in a total of 10 sessions performed twice a week in the period of 45 minutes. The results show that the physiotherapeutic treatment using the hipopressive gymnastics, can promote the strengthening of the pelvic floor, thus improving the quality of life of the individuals researched and reducing the urinary incontinence in physical activity practitioners. 
KEYWORDS: Urinary incontinence, urogynecology physiotherapy, physical activity, hypopressive gymnastics, pelvic floor.

\section{INTRODUÇÃO}

A incontinência urinária (IU) é uma doença de origem multifatorial que atinge milhões de indivíduos, de todas as idades, independente do sexo. A mesma é caracterizada pela perda involuntária de urinaria e pode ocorrer em diversas situações quando a musculatura do assoalho pélvico não é ativada adequadamente, levando a uma alteração na qualidade de vida do indivíduo e gerando desconforto social e higiênico (COSTA; SANTOS, 2012).

O tipo mais comum encontrado é a incontinência urinária de esforço (IUE), definida pela Sociedade Internacional de Continência como a queixa involuntária de perda de urina no esforço físico, tosse ou espirro, sendo identificada pela perda de urina, através da uretra, causada por um aumento da pressão abdominal (MORENO, 2009).

O reflexo da miç̧ão é um reflexo espinhal autônomo, porém pode ser inibido ou facilitado por centros cerebrais, composto pela comunicação dos sistemas nervoso simpático, parassimpático e trato urinário inferior, sendo caracterizado pelas fases de enchimento e esvaziamento da bexiga (MORENO, 2009).

No período da fase de enchimento ocorre o desempenho do sistema simpático, executado pelo nervo hipogástrico, no qual se unindo com os receptores betas da bexiga, ocasionam o relaxamento do músculo detrusor e contração do esfíncter interno da uretra; também quando a bexiga se expande durante o enchimento, são estimulados os receptores proprioceptivos e nociceptivos. Essa mensagem levada pelo nervo pudendo para o centro pontino da micção, onde ocorre a interpretação desse estímulo e o envio desta informação para o nervo pélvico, ocorrendo como resultado a eliminação da urina. Para ter uma micção adequada, as vias neurológicas desde os nervos periféricos até a região medular, devem estar íntegros (MORENO, 2009).

A prática de atividades físicas é algo muito valorizado na atualidade, pela busca na melhoria de qualidade de vida, prevenção e aparência física, sendo 
definida de acordo com Pitanga (2002) como qualquer movimento corporal produzido pelos músculos esqueléticos que resulta em gasto energético maior do que os níveis de repouso.

A fraqueza assoalho pélvico associado a repetição da prática de atividade física, com exercícios de contração abdominal máxima e saltos afetam a pressão intra-abdominal e podem assim gerar a IUE (SILVA et al., 2016).

A fisioterapia uroginecológica atua no tratamento da IU, promover o fortalecimento da musculatura do assoalho pélvico (MAP) e melhorar o quadro de incontinência urinária por esforço. Para a Sociedade Internacional de Continência a fisioterapia uroginecológica é vista como a primeira opção para o tratamento devido ao baixo custo e efetividade na melhora da perda de urina, por meio de recursos terapêuticos, tal como a ginástica hipopressiva (ALENCEAR et al. 2015).

A ginástica hipopressiva é uma técnica desenvolvida pelo fisioterapeuta belga Marcel Caufriez, na década de 50 e adaptada recentemente por outros pesquisadores e tem como característica proporcionar a melhora do tônus da musculatura perineal que se baseia na contração ativa da musculatura abdominal (manobra de aspiração diafragmática) gerando a elevação dos órgãos pélvicos, na direção do diafragma respiratório e respectiva contração supostamente reflexa da musculatura do assoalho pélvico (MAP), assim melhorando o quadro de incontinência urinária (SCARPELINI et al., 2014).

Sob a hipótese da ação da fisioterapia pélvica em pacientes com incontinência urinária, surge o questionamento se a ginástica hipopressiva pode melhorar o fortalecimento da musculatura do assoalho pélvico e melhorar o quadro de incontinência urinária em praticantes de atividade física.

O estudo teve como objetivo geral avaliar a eficácia da ginástica hipopressiva como forma de tratamento para incontinência urinária de esforço em praticantes de atividade física e como objetivos específicos melhorar a força da muscular do assoalho pélvico, diminuir a perda de urina e melhorar a qualidade de vida dos pacientes com incontinência urinária. 


\section{METODOLOGIA}

Foi realizada uma série de casos com duas participantes classificadas com incontinência urinária de esforço, com média de idade $40 \pm 3$ e praticantes de atividade física.

Utilizamos como critério de inclusão participantes com os seguintes requisitos: pacientes de ambos os gêneros, praticantes de atividade física, idade entre 18-45 anos e portador de incontinência urinária por esforço e foram excluídos do estudo, pacientes fora da faixa etária estabelecida, com outro tipo de incontinência urinária, não praticantes de atividade física e déficit de compreensão.

Após a aprovação do Comitê de Ética em Pesquisa (CEP), sob parecer oㅡ 94668918.6.0000.5539 os voluntários assinaram o termo de Consentimento Livre e Esclarecimento (TCLE) e foram submetidos à avaliação individual e invasiva, sendo realizado avaliação Funcional do Aparelho Pélvico (AFA), que determinou o nível de incontinência com uma pontuação; Biofeedback perineal que consiste em um equipamento, que é capaz de fornecer uma resposta visual e/ou sonora para mostrar a força muscular do MAP, e proporcionar diferentes respostas fisiológicas; Pad Test que teve como objetivo avaliar o grau da incontinência urinária, feito com base no peso de um absorvente íntimo; e também o preenchimento do questionário King's Health Questionnaire (KHQ) que avaliou a qualidade de vida.

Para a avaliação de força da musculatura do assoalho pélvico foi utilizada a escala do AFA que é um exame invasivo onde o fisioterapeuta realiza a palpação bidigital no órgão genital e solicita uma contração da musculatura do assoalho pélvico como se fosse interromper o fluxo urinário e o terapeuta avalia a contração, fundamentando na sensibilidade da palpação digital que será classificada em uma escala de 0 a 5 , onde grau 0 indica ausência de função perineal mesmo na palpação; grau 1: contração reconhecida somente na palpação; grau 2: função débil e contração reconhecida na palpação; grau 3: função perineal presente e resistência colocada não é mantida por cinco segundos à palpação; grau 4: função perineal presente e vencendo resistência mantida por mais de cinco segundos por quatro repetições e grau 5: função perineal presente, vencendo uma resistência mantida por cinco segundos e por cinco repetições (ORTIZ et al., 1996). 
Ainda na avaliação foi realizado o Biofeedback manométrico que faz a captação da pressão em milímetros de mercúrio $(\mathrm{mmHg})$, através de uma sonda recoberta por um preservativo lubrificado que é introduzido na cavidade vaginal ou anal do paciente, em seguida é inflada com ar, de modo que desempenhe uma pressão superior à pressão atmosférica. A pressão provocará uma ação no interior da sonda, e a elasticidade do material ajustado ao tônus estático dos tecidos resulta em uma tentativa de se equilibrar. O terapeuta configura o equipamento que faz a captação dos sinais biológicos do paciente, enviando o feedback visual e sonoro para o paciente e o terapeuta. Quando a sonda entra em contato contra as paredes vaginais ou anal, o fisioterapeuta anotará a primeira elevação de pressão (MORENO, 2009).

O Pad Test consiste em um teste que qualifica o grau de incontinência urinária durante a realização de atividades de vida diária. No teste paciaente utiliza um absorvente, previamente pesado por uma balança de precisão. Em seguida ingeri $500 \mathrm{ml}$ de água, permanecendo em repouso por 15 minutos e logo após são requisitadas ações representando atividades de vida diária, como: andar na esteira por 15 minutos, em uma velocidade média de $2,5 \mathrm{Km} / \mathrm{h}$; subir e descer 20 degraus; sentar e levantar 10 vezes; tossir 10 vezes; correr por 1 minuto; pegar objeto no chão e levantar 5 vezes; lavar as mãos em água corrente durante 1 minuto. $\mathrm{Na}$ sequência é retirado o absorvente e novamente pesado, com o objetivo de quantificar a urina perdida durante tais atividades, sendo classificados em perdas leves entre 1 a 10 gramas; perdas moderadas de 11 a 50 gramas, perdas severas: de 51 a 100 gramas e perdas muito severas acima de 100 gramas (ALBUQUERQUE et al., 2011).

Para avaliação do impacto da IU na qualidade de vida foi utilizado o questionário King's Health Questionnaire que consiste em perguntas de assinalar e abrangem a limitações no desempenho de tarefas, limitações físicas/social, problemas de bexiga que afetam no momento, emoções (sono/energia) (MORENO, 2009).

Após a avaliação fisioterapêutica, as pacientes foram submetidas ao tratamento fisioterapêutico com a técnica da Ginástica Hipopressiva realizado, na 
Clínica de Fisioterapia da Unicesumar, totalizando dez sessões, durante 45 minutos, com frequência de duas vezes por semana.

Os exercícios foram realizados em diferentes posições, de forma progressiva de acordo com o grau de dificuldade, levando em consideração a capacidade física e individual de cada paciente.

Nos primeiros atendimentos foram realizados exercícios de baixa complexidade da técnica para adaptação das pacientes. Durante todo o estudo as pacientes foram estimuladas a conscientização da contração e localização da musculatura do assoalho pélvico, inicialmente por meio de posturas mais conhecidas e fáceis para as pacientes, como em pé, com joelhos semi fletidos, anteversão da pelve e alinhamento do tronco. Durante a execução dos movimentos 0 posicionamento das mãos foi intercalado em várias angulações no repetir dos exercícios, inicialmente as mãos e braços a frente do corpo para baixo, em abdução de ombro, cotovelos semi-fletidos e antebraço neutro com punho em pronação, voltados para dentro, depois na altura dos ombros e por fim flexão de ombro $180^{\circ}$.

A partir do $3^{\circ}$ atendimento foram realizados exercícios em posições sentada na bola suíça, com alinhamento de tronco, anteversão da pelve, em diferentes posicionamentos dos braços, na posição de quatro apoios, com retroversão da pelve, alinhamento de tronco e na posição sentada com membro inferior fletido, abduzidos e rotação externa, mãos sobre os joelhos, com alinhamento de tronco.

No 5o atendimento, iniciamos posições com maior duração de tempo de contração, tais como: paciente em cima da cama elástica, com mini band entre os joelhos, sobre a cama elástica levantando intercalando uma perna de cada vez, com anteversão da pelve e alinhamento de tronco, e em ambas posições o intercalar do posicionamento das mãos e na posição de quatro apoios, levantando intercalando uma perna de cada vez. O tempo inicial de contração era de 10 segundos, pacientes evoluíram para 17 segundos nessa etapa.

A partir do $7^{\circ}$ atendimento, iniciamos posições de maior complexidade, nas quais as pacientes já conseguiam realizar a contração por mais tempo, alcançando 20 segundos, tais como: 
Em posição de prancha, posição de ponte levantando intercalado uma perna de cada vez e sentada, com alinhamento de tronco, membro inferior em extensão e dorsiflexão do pé, intercalando o posicionamento das mãos ao repetir o exercício.

No $10^{\circ}$ atendimento, o qual correspondeu ao atendimento final, pacientes conseguiram realizar a contração por 28 segundos, em posições mais complicadas. Como em cima do bozu, com alinhamento de tronco anteversão da pelve e intercalado com posicionamento das mãos com iterar do exercício. Também foi realizado exercício em que as pacientes realizavam em decúbito dorsal, alinhamento de tronco e extensão do membro inferior, realizou o levantamento do mesmo simultaneamente, de início mais alto e diminuindo gradativamente. Outra posição executada foi paciente em decúbito dorsal, flexão de membro inferior, realiza leve elevação da escapula do colchonete, intercalando o posicionamento das mãos durante o exercício.

Foi mantida a mesma ordem na execução dos exercícios em todos os atendimentos, com alinhamento de tronco, retroversão da pelve e posicionamento das mãos como já citados. Em seguida paciente realizava a inspiração, abrindo bem a caixa torácica, após realizava a expiração, soltando todo o ar.

Logo após executava a contração do MAP, intercostais e peitorais, mantida em apneia por alguns segundos cronometrados, seguidos de repouso de 10 segundos. Os exercícios foram repetidos em cada uma das posições por 5 vezes em todos os atendimentos.

O protocolo da ginástica hipopressiva foi composto de treinamento da manobra de aspiração diafragmática que é formada por três movimentos em sequência como contração dos músculos abdominais, contração do MAP pélvico e contração dos músculos intercostais e peitorais.

Ao termino dos atendimentos, as pacientes foram reavaliadas e os dados foram examinados para comprovação dos resultados.

\section{RESULTADO/DISCUSSÃO}


O presente estudo foi realizado com duas participantes, do sexo feminino, paciente $1 \mathrm{C}$. R. O., 43 anos, praticante de treinamento funcional e paciente $2 \mathrm{C}$. M. A., 38 anos, praticante de musculação, ambas com diagnóstico de incontinência urinária de esforço.

Durante a avaliação inicial do assoalho pélvico através do AFA e da palpação, podemos observar que ambas pacientes apresentaram fraqueza da musculatura e após o tratamento fisioterapêutico podemos observar melhora da força muscular, conforme indica a figura 1.

Figura 1: Avaliação do AFA antes e após o tratamento fisioterapêutico.

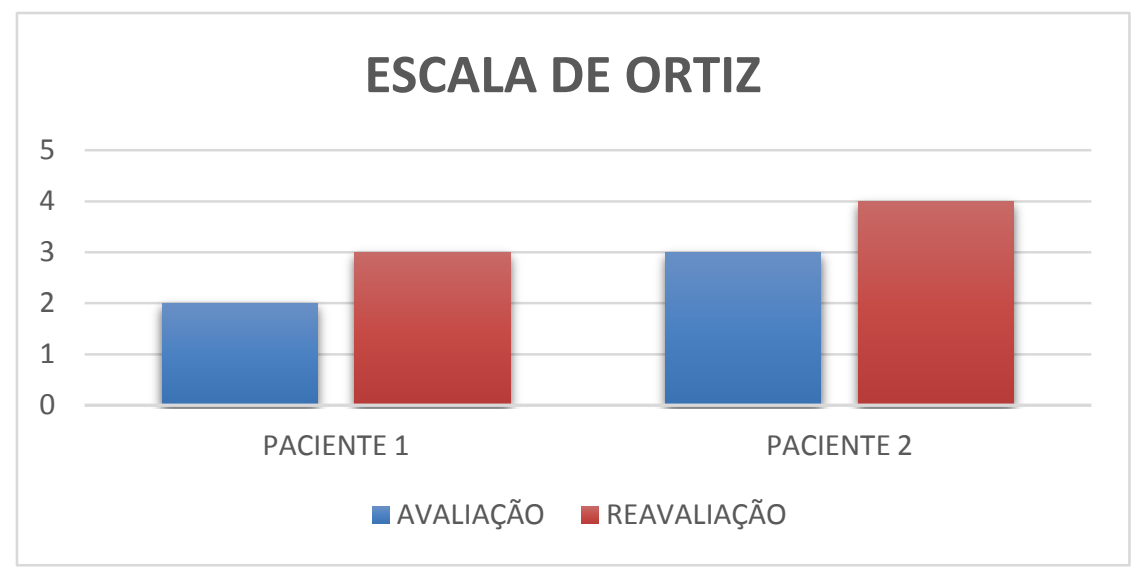

Dados da pesquisa.

Fonte:

A avaliação da força da musculatura de assoalho pélvico foi realizada através do equipamento Biofeedback que registrou os potenciais de ação das contrações do MAP antes e após o tratamento com a ginástica hipopressiva nas duas participantes do estudo, conforme indicado na figura 2. 
Figura 2: Avaliação do Biofeedback antes e após 0 tratamento fisioterapêutico.

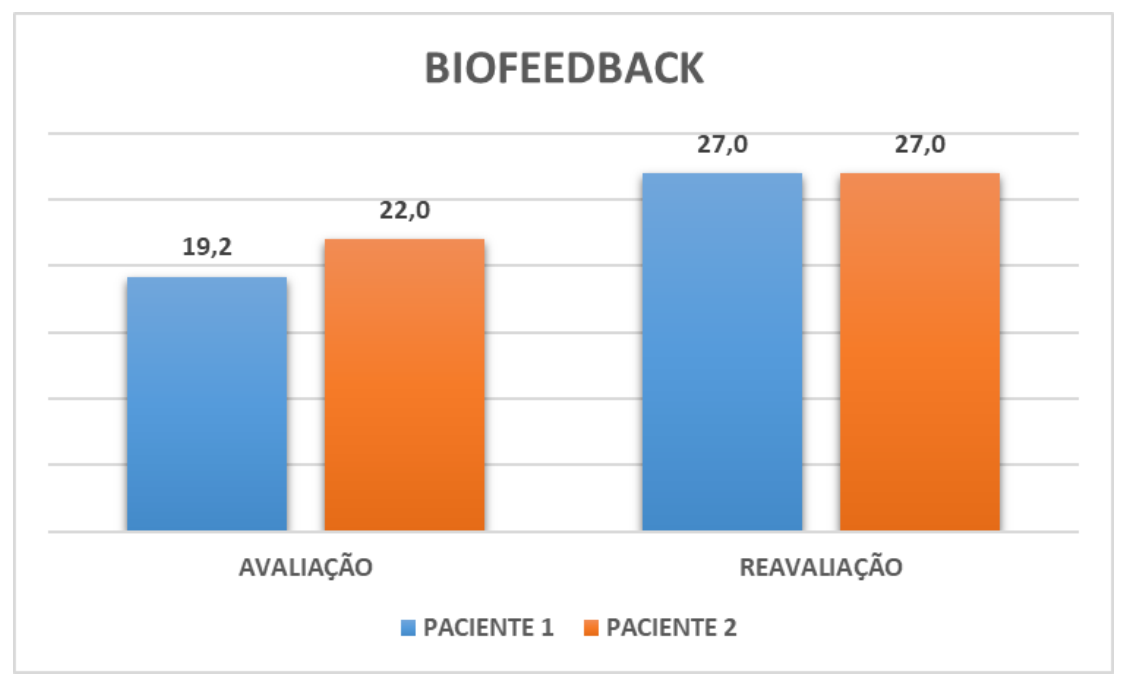

Fonte: Dados da pesquisa.

Já a avaliação do grau de incontinência urinária foi realizada através do Pad Test, onde inicialmente ambas pacientes foram classificadas com perda urinária leve e após o tratamento fisioterapêutico utilizando a ginástica hipopressiva não tiveram mais perdas (figura 3).

Figura 3: Avaliação do PAD TEST antes e após o tratamento fisioterapêutico nas duas participantes da pesquisa

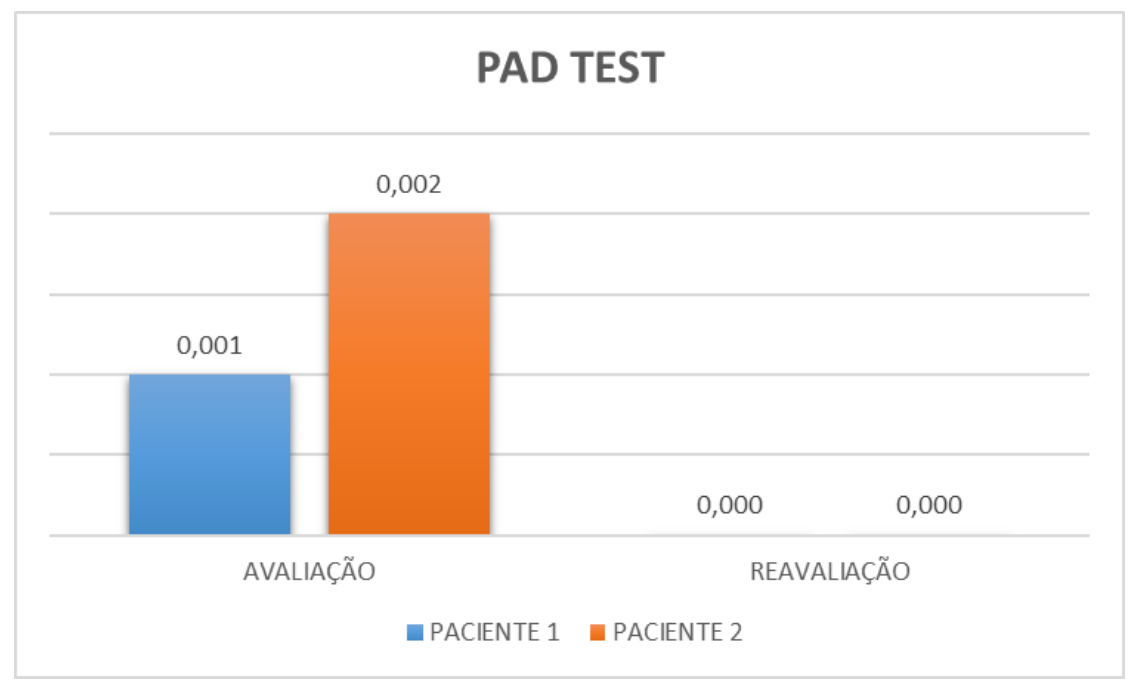

Fonte: Dados da pesquisa. 
Para avaliação do impacto da IU na qualidade de vida, foi aplicado o questionário King's Health Questionnaire (KHQ), através do questionário tornou-se possível a conformação que as pacientes tiveram melhora na qualidade de visa e nas atividades de vida diária.

Após o tratamento fisioterapêutico com 10 sessões, utilizando exercícios baseados na técnica da ginástica hipopressiva, foi possível constatar aumento da força e do tônus muscular do assoalho pélvico e qualidade de vida nas duas pacientes participantes do estudo, que corrobora com os relatos de Scarpelini; et al. (2014), que em seu estudo relataram que a técnica da ginástica hipopressiva promove uma resposta fisiológica através do aumento do tônus e da força muscular. Seleme et al. (2009) reafirma os benefícios da ginastica, relatando que a técnica proporciona uma tonificação perineal e abdominal, a qual possibilita extensa vantagem para pacientes que não têm compreensão ou mostram dificuldade em realizar a contração perineal.

$\mathrm{B} \varnothing$, K. et al. (1988) relatam que mais de $30 \%$ das mulheres não realizam a contração da musculatura do assoalho pélvico e $37 \%$ das mulheres não têm consciência corporal de sua região pélvica, não sendo efetiva a contração desta musculatura. Em função disso Moreno e Mitrano (2004) sugerem a necessidade de um trabalho proprioceptivo sobre o tratamento fisioterapêutico. Da mesma forma Amarenco et al. (1990) defende a importância da conscientização perineal antes de começar qualquer tratamento fisioterapêutico para o assoalho pélvico.

Na pesquisa de Costa et al. (2011) foi utilizada a escala Oxford para qualificar a força dos músculos do assoalho pélvico antes e após o tratamento com ginástica hipopressiva, sendo a média inicial de força muscular do assoalho pélvico de 1,6 $( \pm 0,6)$ e na reavaliação a média final de $2,8( \pm 0,5)$, mostrando assim que a ginástica hipopressiva melhora a ação e força do MAP minimizando tal como observado na presente pesquisa os sintomas de perdas urinárias aos esforços.

Latorre, et al. (2011) relatam em seu estudo que a ginástica hipopressiva promove movimento cranial das vísceras através da ultrassonografia e ressonância magnética. Já a eletromiografia mostrou que no decorrer da manobra de aspiração 
diafragmática acontece uma ativação reflexa da musculatura do assoalho pélvico durante a realização da técnica.

Resultados semelhantes que do presente estudo foram observados por Matheus e Mazzari (2006) ao analisarem e compararem dois grupos para o tratamento de Incontinência Urinária em pacientes que apresentavam grau de contração diminuído e diminuição da estática pélvica, sendo no grupo 1 utilizando cone vaginal e no grupo 2 contração mais respiração. Ambos os grupos associaram exercícios de equilíbrio postural no tratamento. Os resultados revelaram aumento da força muscular em ambos os grupos através do AFA e conscientização da contração do assoalho pélvico, demonstrando efetividade do tratamento no aumento da força muscular perineal.

Já Kakihara (2003) revelou a eficiência da cinesioterapia na recuperação dos sintomas urinários como diminuição da perda urinária pelo aumento da força muscular do MAP, diminuição da frequência miccional, maior intervalo entre as micções, redução do grau de incontinência e maior bem-estar com a qualidade de vida de acordo com os pacientes.

\section{CONSIDERAÇÕES FINAIS}

Pode-se concluir, com a presente pesquisa, que a fisioterapia pélvica utilizando a técnica da ginastica hipopressiva é capaz de melhorar a força e o tônus dos músculos do assoalho pélvico, promovendo assim melhora do quadro de incontinência urinaria de esforço e consequentemente da qualidade de vida. Entretanto, devido à escassez de trabalhos associando a técnica da ginástica hipopressiva com a incontinência urinaria de esforço e ao limitado número de participantes, faz-se necessário a produção de mais estudos com essa relação, para que haja maiores comprovações.

\section{REFERÊNCIAS}

ALBUQUERQUE, M. T. et. al. Correlação entre as queixas de incontinência urinária de esforço e o pad test de uma hora em mulheres na pós-menopausa. Bras. Ginecol. Obstet. Vol.33. Rio de Janeiro Fev. 2011 
ALENCAR, P.D. C.; VENTURA, P. L. Benefícios do treinamento da musculatura do assoalho pélvico no tratamento de mulheres com incontinência urinária de esforço: revisão de literatura. Revista interdisciplinar ciência e saúde, Piauí, p.38-46; Ago/out, 2015

BERBAM, Laura Uttig; DREHER, Daniela Zeni. Exercícios de Kegel e ginástica hipopressiva como estratégia de atendimento domiciliar no tratamento da incontinência urinária feminina: relato de caso (2012-01-17). UNIJUÍ Universidade Regional do Noroeste do Estado do Rio Grande do Sul. Ijuí-RS

CARRERETTE, Fabrício Borges et. al. Incontinência urinária de esforço. Disponível em

http://www.moreirajr.com.br/revistas.asp?id_materia=852\&fase=imprime > acesso em 18 de março de 2018.

COSTA, A.P.; SANTOS, F.D.R.P. Abordagem da fisioterapia no tratamento da incontinência urinária de esforço: revisão da literatura. Revista femina. Rio de Janeiro, maço 2012| vol.40 | no 2. Disponivel em < http://files.bvs.br/upload/S/01007254/2012/v40n2/a3090.pdf > acesso em 20 de março de 2018.

COSTA,T.F, RESENDE, A.P.M, SELEME, M.R., et al. Ginástica Hipopressiva como recurso proprioceptivo para os músculos do assoalho pélvico de mulheres incontinentes. Fisioterapia Brasil, 12 (5): 365-369, 2011.

DINUBILE, N.A. Strength training. Clin. Sports med. American college of sports medicine. 10 (1): 33-62, 1991.

GUEDES, J. M.; PRIGOL, S.; SEBBEN, V.; Prevalência da incontinência urinaria em mulheres praticantes de atividade físicas nas academias da cidade de Erechim. Erechim. v. 38, n.141. 2014.

KAKIHARA, CT. Cinesioterapia na redução da incontinência urinária de pacientes pós-prostatectomizados. Fisioterapia Brasil. 4(4): 265-70, 2003.

KAKIHARA, CT; SENS, YAS; FERREIRA, U. Efeito do Treinamento funcional do assoalho pélvico Associado ou não à Eletroestimulação na Incontinência urinária após prostatectomia radical. Rev. Bras. Fisioter., São Carlos, 11 (6), 2007.

KORELO, Raciele Ivandra Guarda et. al.; Influência do fortalecimento abdominal na função perineal, associado ou não à orientação de contração do assoalho pélvico, em nulíparas ISSN 0103-5150 Fisioter. Mov., Curitiba, v. 24, n. 1, p. 75-85, jan/mar. 2011 licenciado sob uma Licença Creative Commons

LATORRE, G. F. S.; SElEME, M. R.; RESENDE, A. P. M. et al. Ginástica Hipopressiva: As evidências de uma alternativa ao treinamento da musculatura do assoalho pélvico de mulheres com déficit proprioceptivo local. Fisioterapia Brasil, 12 (6): 463-466, 2011.

MORENO, A. L. Fisioterapia em Uroginecologia. $2^{-a}$ edição revisada e ampliada. Barueri, SP: Manole, 2009. 
MORENO AL, Mitrano P. Avaliação do assoalho pélvico: avaliação funcional. In: Moreno AL. Fisioterapia em Uroginecologia. Barueri, SP: Manole; p.107-112, 2004.

ORTINIZ, O. C., NUNEZ, F. C., IBANEZ, G. Evaluación funcional del piso pelviano femenino (clasificación funcional). Bol Soc Latinoameric Urogenecol Cir. Vaginal. 1;5-9, 1996.

PINHEIRO, Brenda de Figueiredo et. al.; Fisioterapia para consciência perineal: uma comparação entre as cinesioterapias com toque digital e com auxílio do biofeedback. ISSN 0103-5150 Fisioter. Mov., Curitiba, v. 25, n. 3, p. 639-648, jul./set. 2012 Licenciado sob uma Licença Creative Commons

PITANGA, Francisco José G. Epidemiologia, atividade física e saúde. Revista Brasileira De Ciência e Movimento, Brasília, v.10, n. 3, p. 49 - 54, 2002.

SCARPELINI, P.; FREITAS, A. O.; SILVA. G. C.; HADDAD. C. A. S.; Protocolo de ginástica hipopressiva no tratamento da incontinência urinaria pós-prostectomia: relato de caso. Revista UNILUS Ensino e Pesquisa Vol. 11 - №. 23 - ISSN 23182083. 2014.

SELEME MR, BERTOTTO A, RIBEIRO VW. Exercícios Hipopressivos. In: PALMA, Paulo César Rodrigues. Urofisioterapia: Aplicações Clínicas das Técnicas Fisioterapêuticas nas Disfunções Miccionais e do Assoalho Pélvico. Campinas/sp: personal link comunicações Itda; p. 295- 306, $2009 .$.

SILVA, G. R. et. al.; Influência de exercícios ativos livres e de alto impacto no fortalecimento da musculatura pélvica. Revista da Universidade Vale do Rio Verde, Três Corações, v. 14, n. 1, p. 393-402, jan./jul. 2016.

TAMANINI, José T. N. et. al.; Validação do "King's Health Questionnaire" para o português em mulheres com incontinência urinária. Revista Saúde Pública, 37(2), p.203-11, 2003. 